\title{
The Association of Clinical Pathologists: 103rd Scientific Meeting
}

The 103rd Scientific Meeting was held in Reading, 5-6 April 1979. Abstracts of the scientific communications follow.

\section{Nutritional megaloblastosis in Asians}

R. P. BRITT AND C. HARPER (Hillingdon Hospital, Hillingdon, Middx) Megaloblastic anaemia has been studied in Asians who have come to Britain from Northern India and East Africa. The majority of subjects have been shown to suffer from a deficiency of vitamin $B_{12}$. In contrast to the indigenous Caucasian patients with megaloblastosis, the majority of Asians have been shown to have no absorption defect. A small number of patients have been found to have classical pernicious anaemia formerly considered rare in Asiatics. Small bowel disease as a cause of vitamin deficiency appears to be rare, and in the majority of patients the disorder appears to have solely a nutritional basis. Evidence is presented indicating that the incidence of nutritional vitamin $\mathbf{B}_{12}$ megaloblastic anaemia has changed considerably in Asians in Britain in the past decade.

\section{Ward measurement of blood glucose}

A. R. W. FORREST (Department of Biochemistry, Royal Infirmary, Glasgow G4 OSF) Blood glucose analyses outside the laboratory are now an accepted part of clinical practice. Clinicians welcome them for their 24-hour availability, their rapidity, and the fact that they do not depend on the cooperation of staff, be they porters or technicians, not under their direct control. However, many clinical chemists have reservations about the ward glucose analysers currently available.

Tonk's criteria suggest that a blood glucose assay should have a precision of around $10-15 \%$ in the normal range if it is to be clinically useful. While few laboratories would be happy if their glucose assays were so imprecise, the successful marketing of instruments operating at this level of precision for ward use shows that clinicians find it acceptable. At present most of these analysers depend on reagent strips such as Dextrostix (Ames Co) or Reflotest (Boehringer Corp.). If the techniques for storing and using the strips are not exactly those laid down by the manufacturers then the results will be dangerously inaccurate.

Two surveys, four months apart, of the ability of the 38 wards and clinics using blood glucose reagent strips at two district general hospitals to analyse a sample for glucose content successfully demonstrated several disturbing features; nursing staff were reluctant to take part, only $25 \%$ of those approached agreeing to participate. One-quarter of the wards produced results so inaccurate that had the samples come from a patient, clinical action based on the results obtained would have been inappropriate. Only one-third of the participants reported their results in SI units, although the local laboratory had been doing so for two years.

The one ward which regularly used any form of quality control and which discussed its routine results with the laboratory produced the best results in both surveys.

In the near future, analysers based on multilayer film technology are likely to appear on the wards. The great potential of these devices for improving patient care will be achieved only if ward and laboratory staff cooperate in their introduction and use.

Electron microscopy of isolated sarcoid type granulomas in Hodgkin's disease

W. JONES WILLIAMS, D. L. JONES, AND J. WHITTAKER (Llandough Hospital, Penarth, and Welsh National School of Medicine, Heath Park, Cardiff, S. Glamorgan) We have studied by electron microscopy sarcoid type granulomas in six patients with Hodgkin's disease. Despite the absence of any evidence of systematic sarcoidosis, the epithelioid and giant cells were identical with those previously described in sarcoidosis and in chronic beryllium disease. The morphology and histochemistry of the cells again suggest that they are functionally synthesising rather than phagocytic cells.

On light microscopy, in 60 patients with Hodgkin's disease diagnosed between 1973 and 1976, we found 11 cases $(16 \%)$ with isolated sarcoid type granulomas. The presence of the granulomas was unrelated to the histological type of Hodgkin's disease, clinical staging or response to treatment.

\section{Centrifugal analysis: is rotor size significant?}

B. T. MARTIN AND G. CANNON (Ninewells Hospital, Dundee) The introduction of centrifugal fast analysers (CFAs) to the range of equipment available to the clinical chemistry laboratory has resulted in much recent interest in their capabilities as replacements for existing obsolescent instrumentation. An apparently fundamental difference among the several systems at present available concerns their relative rotor capacities and the effect these may have on workload processing. We were able to examine this problem during an extensive laboratory evaluation of three commercial CFA systems and found, contrary to expectation, that substantial differences in rotor capacity had only a minor effect on the rate of specimen analysis. 
The instruments used were: Rotochem IIa (Aminco)36 place rotor, CentrifiChem System 400 (Union Carbide) - 30 place rotor, and Gemsaec $3 E$ (Electronucleonics Inc.) - 16 place rotor. The workload examined comprised the daily requests for the enzymes AST, HBD, and $\gamma$-GTP 1 over a one-week period (150 to 200 analyses per day), each day's workload being accumulated and analysed as one continuous sequence. The theoretical capacities of the systems, respectively $210: 175: 135$ enzyme analyses per hour, were found in practice to be $125: 120: 100$. A $50 \%$ reduction in rotor size therefore resulted in a reduction in analysis rate of only $20 \%$, indicating that capacity is not of fundamental importance in a consideration of the merits of these machines. An account of the factors causing this paradox will be given.

Disease activity and IgG turnover in IgG myeloma patients

SUSAN M. WHIPPS, J. WATKINS, F. E. PRESTON, M. ELDRIDGE, AND G. HUDSON (Departments of Haematology and Immunology, University of Sheffield) Serum immunoglobulin levels are frequently used to monitor clinical progress in a variety of disease states. It is frequently forgotten that these serum protein concentrations are not static entities but reflect the dynamic balance between processes such as intra and extra vascular equilibrium and anabolism and catabolism. Consideration of these factors may, therefore, provide the clinician with valuable additional information.

The catabolism of IgG has long been thought of as proportional to its serum concentration (Waldmann and Strober, 1969). Investigations with rheumatoid arthritis patients, however, indicate that it is more closely linked to disease activity, being significantly increased in active disease (Watkins and Swannell, 1973). IgG myeloma patients have also been reported to have increased IgG catabolic rates; these have not always shown a good correlation with serum concentration. Equally, disease status was not always documented (Soloman et al., 1963).

We, therefore, decided to study catabolic rates in IgG myeloma patients possessing very different plasma monoclonal protein concentrations of $\operatorname{IgG}(\mathrm{M})$ and clinical activity.

Immunologically pure IgG was isolated from the serum of myeloma patients by column chromatography on DEAE-cellulose at $\mathrm{pH} 6 \cdot 2$. Each immunoglobulin preparation was labelled with iodine-125 and injected

${ }^{1}$ AST $=$ aspartate aminotransferase $; \quad$ HBD = hydroxybutyrate dehydrogenase; $\gamma$-GTP $=\gamma$-glutamyl transpeptidase intravenously into the 'donor' patient concerned. Elimination of labelled protein was followed by scintillation counting of serum samples at intervals over a minimum period of 28 days. Our preliminary findings (Table) indicated that the IgG plasma half-lives $\left(T \frac{1}{2}\right)$ do not correlate with serum concentrations. It may be important that the asymptomatic patients have normal $T_{\frac{1}{2}}$ values ( $>20$ days) while patients showing clinical deterioration have considerably lower $\mathrm{T}_{\frac{1}{2}}$ values.

\section{References}

Soloman, A., Waldmann, T. A., and Fahey, J. L. (1963). Journal of Laboratory and Clinical Medicine, 62, 1-17.

Waldmann, T. A., and Strober, W. (1969). Progress in Allergy, 13, 1-110.

Watkins, J., and Swannell, A. J. (1973). Annals of the Rheumatic Diseases, 32, 247-250.

Platelet monoamine oxidase activity as a diagnostic aid in megaloblastic anaemia?

VIVETTE GLOVER, M. SANDLER (Queen Charlotte's Hospital, London) and A. HUGHES, A. V. HOFFBRAND (Royal Free Hospital, London) Platelet monoamine oxidase activity has been measured in 17 patients with untreated megaloblastic anaemia due to vitamin $\mathbf{B}_{\mathbf{1 2}}$ or folate deficiency and in 20 normal subjects. The levels were more than doubled in megaloblastic anaemia $(32.0 \pm 1.2 \mathrm{nmol}$ phenylethylamine oxidised $/ \mathrm{h} / \mathrm{mg}$ protein) compared with a normal population $(15.0 \pm 3.8 \mathrm{nmol}$ phenylethylamine oxidised/h/mg protein) $(\mathrm{P}<0.001)$. Values fell to normal in two patients when retested three months after starting vitamin $B_{12}$ therapy. Enzyme activity in the patient group was significantly correlated with the severity of the disease as determined by the deoxyuridine-suppression test $(P<0.025)$ and marrow morphology $(P<0.05)$. Low serum vitamin $B_{12}$ or folate were not in themselves related to the increase. The ease of measuring platelet MAO activity and the lack of trauma to the patient compared with a marrow biopsy suggests that in some circumstances it could be a useful diagnostic test.

It has previously been shown that platelet MAO activity is partly under genetic control. There is evidence to suggest that platelet maturity may be another factor that can control its specific activity.

Laboratory investigations of infectious keratoconjunctivitis

L. A. HATCH (University of Western Ontario, Canada) The scrapings, swabbings, and cytological smears collected

\begin{tabular}{|c|c|c|c|c|c|}
\hline Status & 'M' type & $\begin{array}{l}\text { Total IgG } \\
\text { conc }\end{array}$ & $\begin{array}{l}\text { 'M' conc } \\
(g / I)\end{array}$ & $\begin{array}{l}\text { Fract. catabolic } \\
\text { rate }\end{array}$ & $T_{\frac{1}{2}}($ days $)$ \\
\hline $\begin{array}{l}\text { BS Deteriorating } \\
\text { BG Asymptomatic } \\
\text { IG Asymptomatic } \\
\text { VC Asymptomatic } \\
\text { ME Deteriorating }\end{array}$ & $\begin{array}{l}\mathbf{G} \lambda \\
\mathbf{G} \kappa \\
\mathbf{G} \kappa \\
\mathbf{G} \kappa \\
\mathbf{G} \kappa\end{array}$ & $\begin{array}{r}14 \\
26 \\
8 \\
15 \\
32\end{array}$ & $\begin{array}{c}10 \\
26 \\
<0 \cdot 1 \\
8 \\
32\end{array}$ & $\begin{array}{l}0.063 \\
0.025 \\
0.028 \\
0.028 \\
0.069\end{array}$ & $\begin{array}{l}11 \\
28 \\
25 \\
25 \\
10\end{array}$ \\
\hline
\end{tabular}

This work was supported by the Yorkshire Cancer Research Campaign. 
from patients with acute, subacute, and chronic keratoconjunctivitis over several years were examined bacteriologically and for the presence of viruses. These findings are presented in illustration of the relative commonness of virus infections and the superiority of cytological examination by Giemsa stain over Gram stain in providing rapid information to the attending physicians concerning the aetiology of the infection and indicating appropriate treatment.

Supporting evidence for 'de novo' pyrimidine synthesis in pernicious anaemia under treatment

T. E. PARRY (Department of Haematology, Llandough Hospital, South Glamorgan Area Health Authority (Teaching)) The subnormal serum uracil levels reported in pernicious anaemia in relapse and their return to normal values or above by vitamin $B_{12}$ even in physiological doses $(2 \mu \mathrm{g} /$ day $)$ are the exact antithesis of the methylation of dUMP to dTMP being the main biochemical defect in vitamin $\mathbf{B}_{12}$ deficiency. They strongly suggest, however, that 'de novo' pyrimidine synthesis is induced in pernicious anaemia by vitamin $B_{12}$. Aspartic acid is an early pyrimidine precursor in the only synthetic pathway known. This amino acid has been assayed microbiologically both before and for five days after treatment with a single dose of $200 \mu \mathrm{g}$ vitamin $B_{12}$ in 10 cases of pernicious anaemia in whom the serum uracil levels have previously been reported. There was a sharp fall in the serum aspartic acid level in six of the 10 cases in the first 24 hours and a similar fall in two other cases in 48 hours (mean pre-treatment level $61 \cdot 62 \mu \mathrm{mol} / \mathrm{l}$, SD 10.14, mean level after 24 hours $49.9 \mu \mathrm{mol} / 1, P<0.025)$. The same change was produced by vitamin $\mathrm{B}_{12}$ in a physiological dose of $2 \mu \mathrm{g} /$ day. The fall in aspartic acid precedes the rise in serum uracil level and reticulocyte count by approximately 72 and 96 hours respectively. It also precedes by 48 hours the rise in serum methionine level previously reported in nine other cases of pernicious anaemia studied under similar conditions. The fall in serum aspartic acid is, therefore, a very early change induced by vitamin $B_{12}$ and occurs at a time when megaloblastic changes are being reversed in the marrow, that is, when changes in DNA metabolism are known to occur and before any changes in protein synthesis are recognised. Both the observed fall in the serum aspartic acid and its timing are, therefore, consistent with 'de novo' pyrimidine synthesis being induced by vitamin $B_{12}$.

\section{Chloramphenicol and the neonate}

ANNE MUlhall AND J. DE Louvois (Department of Microbiology, Queen Charlotte's Maternity Hospital) Although it is associated with significant potential toxicity, chloramphenicol is the antibiotic of choice in many cases of Gram-negative neonatal meningitis, $30-50 \%$ of serum levels being attainable in the cerebrospinal fluid.

During the neonatal period, especially in preterm infants, the microsomal enzyme systems responsible for chloramphenicol metabolism are not fully developed. In addition, glomerular filtration, the route of excretion of active chloramphenicol (the form thought to cause toxicity), is functioning at only $30-50 \%$ of adult levels. Other drugs, in particular barbiturate, may accelerate the metabolism of chloramphenicol by induction of hepatic enzymes. If the special circumstances of neonatal metabolism are ignored, it is possible for subtherapeutic or toxic concentrations of chloramphenicol to occur. There is, therefore, an obvious necessity for monitoring chloramphenicol levels during therapy in the neonate.

A new method of plate diffusion assay utilising $0.5 \%$ yeast extract agar and $S$. lutea (NCTC 8340) was used to study chloramphenicol therapy in 13 term and 11 premature infants with meningitis. Term infants over 1 week of age consistently had lower levels of chloramphenicol in both serum and cerebrospinal fluid when compared with premature infants of less than 34 weeks' gestation.

Almost all of the term infants had serum levels of chloramphenicol below $10 \mathrm{mg} / 1 \mathrm{l}$ hour after injection, and in many instances cerebrospinal fluid samples from this group contained no detectable chloramphenicol. This may have been due in part to the dosage regimens employed. Preterm infants received an average $43 \pm \mathrm{mg} / \mathrm{kg} /$ day (range $22-75 \mathrm{mg} / \mathrm{kg} /$ day) while term infants received $40 \pm \mathrm{mg} / \mathrm{kg} /$ day (range $17-80 \mathrm{mg} / \mathrm{kg} /$ day). The currently recommended dosage for term infants over 1 week old is $50 \mathrm{mg} / \mathrm{kg} /$ day while that for preterm and term infants under 1 week old is $25 \mathrm{mg} / \mathrm{kg} /$ day. In the patients studied, chloramphenicol was therefore being given at subtherapeutic doses in many cases.

Electron microscopic structure of human cilia: what is normal?

B. FOX AND T. B. BULL (Department of Histopathology, Charing Cross Hospital Medical School, London) Recently there has been an increasing number of reports of abnormalities in the ultramicroscopic structure of cilia, particularly since Eliasson et al. (1977) described a syndrome of congenital immotility of cilia and spermatozoa with absence of dynein arms, associated with chronic airways disease. Other cilial abnormalities described are variations in the numbers of microtubules (McDowell et al., 1976), alterations in the position of the central two singlet microtubules (Sturgess, 1978), and presence of compound cilia (Friedmann and Bird, 1971; Ailsby and Ghadially, 1973; Lupin and Misko, 1978).

In a recent investigation of possible cilial abnormalities in retinitis pigmentosa (Arden and Fox, 1978), it became apparent that there was a need to establish the normal electron microscopic appearances of cilia in humans. We therefore examined cilia from the nasal mucosa of the inferior turbinate of the nose in 11 controls. An average of about 20 transverse sections of cilia were counted in which the microtubular structure was clearly seen. Use of a goniometer stage made it easier to obtain true transverse sections. Although most of the cilia showed the classical $9+2$ microtubular pattern in the axoneme in about $3 \%$ of cilia $(\mathrm{N}=11 ; \bar{X}=3.55 \% ; \mathrm{SE}=0.84 \%)$ the microtubular pattern was abnormal. There was an increase or decrease in the number of outer doublets, occasional accessory singlets, and only one central singlet instead of the normal two. Dynein arms were completely absent in $4 \%$ of cilia; in the rest there was an 
average of 7-8 dynein arms per cilium $(\mathrm{N}=9 ; \bar{X}=28.98 \%$; $\mathrm{SE}=2.53 \%$; taking 18 dynein arms per cilium as $100 \%$ ). We excluded two of the controls because they had bronchiectasis, a condition in which alteration in the number of dynein arms has been described (Eliasson et al., 1977). Compound cilia are known to occur in chronic sinusitis, allergic rhinitis, and bronchiectasis (Friedmann and Bird, 1971; Lupin and Misko, 1978) so we excluded six of the controls in assessing the number of compound cilia present. In this smaller group of controls $(\mathrm{N}=5)$ no compound cilia were found.

\section{References}

Ailsby, R. L., and Ghadially, F. N. (1973). Journal of Pathology, 109, 75-78.

Arden, G. B., and Fox, B. (1978). Personal observations.

Eliasson, R., et al. (1977). New England Journal of Medicine, 297, 1-6.

Friedmann, I., and Bird, E. S. (1971). Laryngoscope, 81, 1852-1868.

Lupin, A. J., and Misko, G. J. (1978). Journal of Otolaryngology, 7, 95-102.

McDowell, E. M., et al. (1976). Archives of Pathology and Laboratory Medicine, 100, 429-436.

Sturgess, J. (1978). Journal of Cell Biology, 79, 298a. 